\title{
Laminar dispersion in parallel plate sections of flow systems used in analytical chemistry and chemical engineering
}

\author{
Spas D. Kolev ${ }^{1, *}$ and Willem E. van der Linden \\ Laboratory for Chemical Analysis, Department of Chemical Technology, University of Twente, P O Box 217, \\ NL-7500 AE Enschede (The Netherlands)
}

(Received 6th November 1990)

\begin{abstract}
An exact solution of the convective-diffusion equation for fully developed parallel plate laminar flow was obtanned It allows the derivation of theoretical relationships for calculating the Peclet number in the axially dispersed plug flow model and the concentration distribution perpendicular to the direction of the flow, provided that the corresponding solution of this model is known. The convective-diffusion equation was solved numerically using the implicit alternatıng-direction finite difference method It was found that the theory developed is valid for Fourier numbers greater than 1.0. The results obtaned can be used for the mathematical modelling of parallel plate process heat and mass exchangers, haemodialysers and flow-injection and contınuous-flow manifolds with on-line dialysis units with parallel plate geometry.
\end{abstract}

Keywords Flow system, Lamınar dispersıon, Mathematıcal modellıng, Parallel plate laminar flow

Parallel plate flow can be encountered in numerous types of apparatus used in different areas (e.g., process mass- and heat-exchange devices $[1,2]$, parallel plate haemodialysers used in medicine [3-7] and on-line dialysers incorporated in manifolds for segmented [8] and unsegmented [9] contınuous-flow analysis [10-13]). The effectiveness of the overall transfer process in all these devices depends to a great extent on their flow pattern. For that reason its correct mathematical description is of great importance in the development of an adequate general model. In process flow systems both laminar and turbulent flow can be observed whereas in the medical and analytical

\footnotetext{
1 Permanent address Faculty of Chemistry, University of Sofia, Anton Ivanov Ave 1, BG-1126 Sofia, Bulgaria.
}

on-line dialysers the flow conditions characterized by the Reynolds number show that laminar flow should prevail.

The mathematical description of the flow pattern based on the Navier-Stokes equations [2], if possible at all, is associated with considerable computational difficulties even in apparatus with pure laminar flow and with the simplest geometry, i.e., parallel plate or tubular. A simplified approach which has been used successfully in chemical engineering for the mathematical description of all patterns of flow other than plug flow and back-mixing is the introduction of the so-called hydraulic models $[14,15]$. Some of the most important parameters of the parallel plate flow systems (e.g., concentration, linear flow-rate) vary considerably from point to point. For that reason, the distributed parameter hydraulic models seem 
to be more appropriate than the lumped-parameter models (e.g., Ideally mixed tank model), although the latter type are simpler from a mathematical point of view.

Among the distributed-parameter hydraulic models, the axially dispersed plug flow model, which is the simplest representative of a large class of dispersion models, has found wide application in the mathematical modelling of both process and analytical flow-through systems [16-24]. Its main advantages over other models of this class are its relative mathematical simplicity and the existence of some theoretical and empirical equations for the calculation of its only parameter, i.e., the axial dispersion coefficient. For that reason it seems logical to try to describe the flow pattern in parallel plate dialysers with the help of the axially dispersed plug flow model. This will allow the use of the mathematical models of traditional singleline flow-injection systems based on the hydraulic model mentioned above [16-24] for the description of the more complicated systems with dialysis modules.

The aim of this paper is to describe the dispersion in parallel plate flow with fully developed laminar velocity profile in terms of the axially dispersed plug flow model.

\section{THEORETICAL CONSIDERATIONS}

The dispersion in a fully developed circular laminar flow has been extensively investigated, both theoretically and experimentally. A relatively simple relationship was derived by Taylor $[25,26]$ for the calculation of the axial dispersion coefficient if the dispersion process is diffusion controlled and the axial diffusion is negligible. Aris [27] extended this equation for the case when the axial diffusion must be taken into account. A further generalization of this approach was introduced by Gill $[28,29]$ by assuming a time dependence for the axial dispersion coefficient. Ananthakrishnan et al. [30] determined the region of applicability of the Taylor-Aris theory using as criteria the Peclet number based on the tube diameter and "the reduced to molecular diffusion scale mean residence time", known in chemical en- gineering as the Fourier number. These results made possible the theoretical calculation of the axial dispersion coefficient of various flow-injection systems which otherwise can be determined only experimentally using the stimulus-response technique [22].

An approach similar to that of Gill $[28,29]$ was utilized in this investigation for the calculation of the axial dispersion coefficient (Peclet number) in the case of laminar parallel plate flow. For fully developed parallel plate laminar flow the diffusion-convection equation describing the spatial and temporal distribution of the solute in such a flow has the following form (all symbols are defined in Table 1):

$$
\frac{\partial c}{\partial t}+u_{0}\left[1-\left(\frac{y}{a}\right)^{2}\right] \frac{\partial c}{\partial x}-D_{\mathrm{m}}\left(\frac{\partial^{2} c}{\partial x^{2}}+\frac{\partial^{2} c}{\partial y^{2}}\right)=0
$$

where $c$ is the concentration, $t$ is the time, $x$ and $y$ are the space coordinates, $a$ is half of the distance between the two plates, $D_{\mathrm{m}}$ is the molecular diffusion coefficient and $u_{0}$ is the maximum linear flow-rate at $y=0$. The average linear flowrate between the two plates $1 \mathrm{~s}$ equal to $\frac{2}{3} u_{0}$. The flow is symmetrical with respect to the central plane at $y=0$.

The initial and boundary conditions in the case of step-function input at $x=0$ are

$$
\begin{aligned}
& c(0, x, y)=0(x>0) \\
& c(t, 0, y)=c_{0}(t>0) \\
& c(t, \infty, y)=0 \\
& \left(\frac{\partial c}{\partial y}\right)_{y=0}=\left(\frac{\partial c}{\partial y}\right)_{y=a}=0
\end{aligned}
$$

If a new axial coordinate moving with the average velocity of flow is defined as

$x_{1}=x-\frac{2}{3} u_{0} t$

and dimensionless quantities and varuables are introduced, Eqn. 1 and its initial and boundary conditions become

$$
\begin{aligned}
& \frac{\partial C}{\partial \theta}+\left(\frac{1}{2}-\frac{3}{2} Y^{2}\right) \frac{\partial C}{\partial X_{1}}-\tau \beta\left(\frac{\partial^{2} C}{\partial X_{1}^{2}}\right)-\tau\left(\frac{\partial^{2} C}{\partial Y^{2}}\right) \\
& \quad=0
\end{aligned}
$$




\section{TABLE 1}

Symbols and definitions ${ }^{\text {a }}$

\begin{tabular}{|c|c|}
\hline Symbol & Defrnition \\
\hline$a$ & $\begin{array}{l}\text { Half of the distance between the parallel plates } \\
\text { (m) }\end{array}$ \\
\hline$c$ & Concentration $\left(\mathrm{mol} \mathrm{m}^{-3}\right)$ \\
\hline$c_{0}$ & Intial sample concentration $\left(\mathrm{mol} \mathrm{m}^{-3}\right)$ \\
\hline$C$ & $=c / c_{0}$. Dimensionless concentration \\
\hline$D_{L_{t}}$ & Axial dispersion coefficient $\left(\mathrm{m}^{l} \mathrm{~s}^{-1}\right)$ \\
\hline$D_{\mathrm{m}}$ & Molecular diffusion coefficient $\left(\mathrm{m}^{2} \mathrm{~s}^{-1}\right)$ \\
\hline$f_{k}$ & Coefficients defıned in Eqn. 3 \\
\hline$L$ & Characteristıc length (m) \\
\hline $\mathrm{Pe}_{\mathrm{t}}$ & $=u L^{\prime-1} / D_{L_{t}}$ Peclet number \\
\hline$t$ & time $(s)$ \\
\hline$u$ & Average linear flow rate $\left(\mathrm{m} \mathrm{s}^{-1}\right)$ \\
\hline$u_{0}$ & $=1.5 u$. Maxımum linear flow-rate $\left(\mathrm{m} \mathrm{s}^{-1}\right)$ \\
\hline$x$ & Axıal conrdınate $(\mathrm{m})$ \\
\hline$x_{1}$ & $=x-u t$. Transformed axial coordinate \\
\hline$X$ & $=x / L$ Dimensionless axıal coordınate \\
\hline$X_{1}$ & $\begin{array}{l}=x_{1} / L . \text { Dimensionless transformed axial coordi- } \\
\text { nate }\end{array}$ \\
\hline$y$ & Coordinate perpendicular to the parallel plates (m) \\
\hline$Y$ & $\begin{array}{l}=y / a \text { Dimensionless coordinate perpendicular to } \\
\text { the parallel plates }\end{array}$ \\
\hline$\alpha$ & $\begin{array}{l}\text { Dimensionless time of injection (for time injection) } \\
\text { or dimensionless length of the initial sample plug } \\
\text { (for slug injection) }\end{array}$ \\
\hline$\beta$ & $=(a / L)^{2}$. Geometrical dimensionless group \\
\hline$\theta$ & $=t u / L$ Dimensionless tıme \\
\hline$\tau$ & $=D_{\mathrm{m}} L /\left(u a^{2}\right)$. Founer number \\
\hline
\end{tabular}

${ }^{a}$ Subscripts step, tıme and slug refer to step, tıme and slug injection, subscript $\mathrm{m}$ refers to average concentration in the cross-section of the flow.

$$
\begin{aligned}
& C(0, X, Y)=0(X>0) \\
& C(\theta, 0, Y)=1(\theta \geqslant 0) \\
& C(\theta, \infty, Y)=0 \\
& \left(\frac{\partial C}{\partial Y}\right)_{Y=0}=\left(\frac{\partial C}{\partial Y}\right)_{Y=1}=0
\end{aligned}
$$

where $C=c / c_{0}, \quad X_{1}=x_{1} / L, \quad Y=y / a, \quad \tau=$ $D_{\mathrm{m}} L / u a^{2}, \beta=(a / L)^{2}$ and $\theta=t u / L$. The concentration is detected at $x=L$ and for that reason $L$ is assumed to be the characteristic length of the flow system.

The concentration can be expanded in $X_{1}$ in the following way:

$$
C=C_{\mathrm{m}}+\sum_{k=1}^{k=\infty} f_{k}(\theta, Y)\left(\frac{\partial^{k} C_{\mathrm{m}}}{\partial X_{1}^{k}}\right)
$$

where $C_{\mathrm{m}}$ is the mean concentration in a crosssection of the flow, defined as:

$$
C_{\mathrm{m}}=\int_{0}^{1} C \mathrm{~d} Y
$$

If $C$ in Eqn. 2 is replaced by the corresponding expansion (Eqn. 3), the resulting equation will be

$$
\begin{aligned}
& \frac{\partial C_{\mathrm{m}}}{\partial \theta}+\left(\frac{1}{2}-\frac{3}{2} Y^{2}\right) \frac{\partial C_{\mathrm{m}}}{\partial X_{1}}-\tau \beta\left(\frac{\partial^{2} C_{\mathrm{m}}}{\partial X_{1}^{2}}\right) \\
& +\sum_{k=1}^{k=\infty}\left\{\left[\frac{\partial f_{k}}{\partial \theta}-\tau\left(\frac{\partial^{2} f_{k}}{\partial Y^{2}}\right)\right] \times \frac{\partial^{k} C_{\mathrm{m}}}{\partial X_{1}^{k}}\right. \\
& +\left(\frac{1}{2}-\frac{3}{2} Y^{2}\right) f_{k}\left(\frac{\partial^{k+1} C_{\mathrm{m}}}{\partial X_{1}^{k+1}}\right)-\tau \beta f_{k}\left(\frac{\partial^{k+2} C_{\mathrm{m}}}{\partial X_{1}^{k+2}}\right) \\
& \left.+f_{k}\left(\frac{\partial^{k+1} C_{\mathrm{m}}}{\partial \theta \partial X_{1}^{k}}\right)\right\}=0
\end{aligned}
$$

The well known axially dispersed plug flow model

$\frac{\partial C_{m}}{\partial \theta}=\frac{1}{P e} \times \frac{\partial^{2} C_{m}}{\partial X^{2}}-\frac{\partial C_{m}}{\partial X}$

can be generalized with respect to its axial dispersion term

$$
\frac{\partial C_{\mathrm{m}}}{\partial \theta}=\sum_{i=1}^{k=\infty} \frac{1}{P e_{i}(\theta)} \times \frac{\partial^{\imath} C_{\mathrm{m}}}{\partial X^{i}}-\frac{\partial C_{\mathrm{m}}}{\partial X}
$$

where the time-dependent Peclet numbers are defined with respect to the characteristic length $(L)$ of the flow system:

$$
P e_{t}=u L^{t-1} / D_{L_{l}} \quad l=1,2,3, \ldots
$$

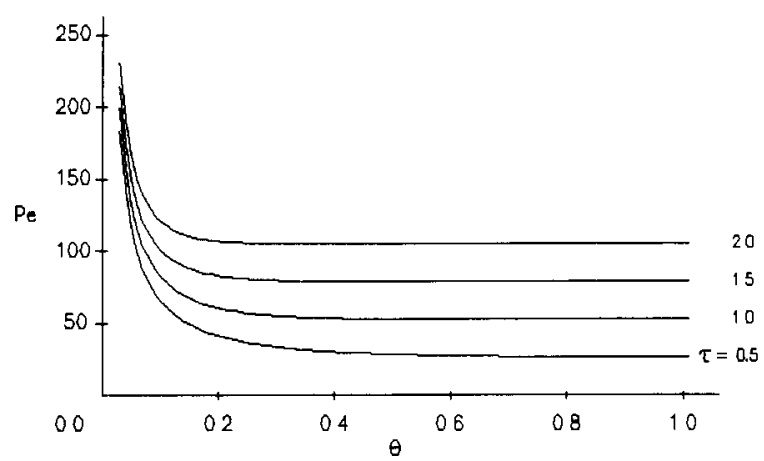

Fig 1 Dependence of $\mathrm{Pe}_{2}$ on the dimensionless time, $\theta$, for different values of the Fourier number, $\tau$ 
If it is assumed, as in the previous considerations, that the coordinate system moves with the mean speed of flow (i.e., $X_{1}=X-\theta$ ), Eqn. 7 is transformed into an equation similar to Fick's second law (Eqn. 9):

$\frac{\partial C_{\mathrm{m}}}{\partial \theta}=\sum_{t=1}^{t=\infty} \frac{1}{P e_{t}} \times \frac{\partial^{i} C_{\mathrm{m}}}{\partial X_{1}^{i}}$

If $\partial C_{\mathrm{m}} / \partial \theta$ in Eqn. 5 is replaced with the corresponding expression from Eqn. 9, the following equation is obtained:

$$
\begin{aligned}
& \sum_{i=1}^{i=\infty} \frac{1}{P e_{\imath}} \times \frac{\partial^{\imath} C_{\mathrm{m}}}{\partial X_{1}^{i}}+\left(\frac{1}{2}-\frac{3}{2} Y^{2}\right) \frac{\partial C_{\mathrm{m}}}{\partial X_{1}}-\tau \beta\left(\frac{\partial^{2} C_{\mathrm{m}}}{\partial X_{1}^{2}}\right) \\
& +\sum_{k=1}^{k=\infty}\left\{\left[\frac{\partial f_{k}}{\partial \theta} \tau\left(\frac{\partial^{2} f_{k}}{\partial Y^{2}}\right)\right] \times \frac{\partial^{k} C_{\mathrm{m}}}{\partial X_{1}^{k}}\right. \\
& +\left(\frac{1}{2}-\frac{3}{2} Y^{2}\right) f_{k}\left(\frac{\partial^{k+1} C_{\mathrm{m}}}{\partial X_{1}^{k+1}}\right)-\tau \beta f_{k}\left(\frac{\partial^{k+2} C_{\mathrm{m}}}{\partial X_{1}^{k+2}}\right) \\
& \left.+f_{k} \sum_{i=1}^{i=\infty} \frac{1}{P e_{l}}\left(\frac{\partial^{k+\imath} C_{\mathrm{m}}}{\partial X_{1}^{k+\imath}}\right)\right\}=0
\end{aligned}
$$

Equation 10 will be satisfied if all coefficients of $\partial^{k} C_{\mathrm{m}} / \partial X_{1}^{k}$ are equal to zero. An infinite set of partial differential equations is generated:

$$
\begin{aligned}
& \frac{\partial f_{1}}{\partial \theta}=\tau\left(\frac{\partial^{2} f_{1}}{\partial Y^{2}}\right)-\left(\frac{1}{2}-\frac{3}{2} Y^{2}+\frac{1}{P e_{1}}\right) \\
& \frac{\partial f_{2}}{\partial \theta}=\tau\left(\frac{\partial^{2} f_{2}}{\partial Y^{2}}\right)-\left(\frac{1}{2}-\frac{3}{2} Y^{2}+\frac{1}{P e_{1}}\right) f_{1}+\tau \beta-\frac{1}{P e_{2}}
\end{aligned}
$$

$$
\begin{aligned}
& \frac{\partial f_{k}}{\partial \theta}= \tau\left(\frac{\partial^{2} f_{k}}{\partial Y^{2}}\right)-\left(\frac{1}{2}-\frac{3}{2} Y^{2}+\frac{1}{P e_{1}}\right) f_{k-1} \\
&+\left(\tau \beta-\frac{1}{P e_{2}}\right) f_{k-2}-\sum_{t=3}^{t=k}\left(\frac{1}{P e_{t}}\right) f_{k-t} \\
&(k=3,4,5, \ldots)
\end{aligned}
$$

where $f_{0}=1$.

From the initial and boundary conditions of Eqn. 2, it follows that

$$
\begin{aligned}
& f_{k}(0, Y)=0 \\
& \left(\frac{\partial f_{k}}{\partial Y}\right)_{Y=0}=\left(\frac{\partial f_{k}}{\partial Y}\right)_{Y=1}=0
\end{aligned}
$$

Equation (4) requires

$\int_{0}^{1} f_{k} \mathrm{~d} Y=0$

By integrating Eqn. 11 with respect to $Y$ from 0 to 1 it can be shown that $P e_{1} \rightarrow \infty$ (i.e., $D_{L_{1}}=0$ ). The same approach gives for the remainder of the Peclet numbers the following equations:

$$
\begin{aligned}
& \frac{1}{P e_{2}}=\tau \beta+\frac{3}{2} \int_{0}^{1} f_{1} Y^{2} \mathrm{~d} Y \\
& \frac{1}{P e_{k}}=\frac{3}{2} \int_{0}^{1} f_{k-1} Y^{2} \mathrm{~d} Y \quad(k=3,4,5, \ldots)
\end{aligned}
$$

From Eqns. 15 and 16, it follows that for calculating the Peclet numbers in Eqns. 9 and 10 all functions $f_{k}$ must be determined explicitly.

Equation 11 was solved by Laplace transforms:

$$
\begin{aligned}
f_{1}= & \frac{1}{4 \tau}\left(-\frac{7}{30}+Y^{2}-\frac{Y^{4}}{2}\right)-\frac{6}{\pi^{4} \tau} \sum_{k=1}^{k-\infty}(-1)^{k} \\
& \times\left[\frac{\cos (\pi k Y)}{k^{4}}\right] \exp \left(-\pi^{2} k^{2} \tau \theta\right)
\end{aligned}
$$

After substitutıng $f_{1}$ in Eqn. 15, the following relationship for $1 / \mathrm{Pe}_{2}$ was obtained:

$$
\begin{aligned}
\frac{1}{P e_{2}}= & \tau \beta+\frac{2}{105} \times \frac{1}{\tau}-\frac{18}{\pi^{6} \tau} \sum_{k=1}^{k=\infty} \frac{1}{k^{6}} \\
& \times \exp \left(-\pi^{2} k^{2} \tau \theta\right)
\end{aligned}
$$

In Fig. 1 it can be seen that for $\tau$ values greater than 1.0 the Peclet number is virtually independent of time for $\theta$ values greater than 0.2 . In fact, the sample plug reaches the detection point at $X=1$ at $\theta$ values much greater than 0.2 and for this reason the steady-state part of $1 / \mathrm{Pe}_{2}$ equal to $\tau \beta+2 /(105 \tau)$ can be used instead of Eqn. 18. For most of the parallel plate laminar flow systems encountered in practice, $\tau \beta$ is much smaller than $2 /(105 \tau)$ and therefore can be neglected. In fact, if both sides of Eqn. 18 are multiplied by $u L$ it can be seen that $\tau \beta$ is the axial diffusion component of the axial dispersion coefficient $\left(D_{L}\right)$ in the classical axially dispersed plug flow model:

$$
\begin{aligned}
D_{L}= & D_{\mathrm{m}}+\frac{2}{105}\left(\frac{u^{2} a^{2}}{D_{\mathrm{m}}}\right)-\frac{18}{\pi^{6}}\left(\frac{u^{2} a^{2}}{D_{\mathrm{m}}}\right) \sum_{k=1}^{k=\infty} \frac{1}{k^{6}} \\
& \times \exp \left(-\pi^{2} k^{2} \tau \theta\right)
\end{aligned}
$$


In principle, all $f_{k}$ functions can be determined completely but this is a tedious task and in addition, as will be seen later, their effect on the overall concentration distribution is insignificant. The latter can be proved if the steady-state parts of the $f_{k}$ functions are compared. They can easily be determined by integration of Eqns. 12 and 13, provided that $\partial f_{k} / \partial \theta=0$. The unknown integrational constants can be determined from conditions $14 \mathrm{~b}$ and $14 \mathrm{c}$. The equations for $f_{2}$ and $f_{3}$ are $f_{2}=\frac{1}{32 \tau^{2}}\left(-\frac{29}{6300}-\frac{17}{105} Y^{2}+\frac{17}{30} Y^{4}-\frac{7}{15} Y^{6}+\frac{3}{28} Y^{8}\right)$

$$
\begin{aligned}
f_{3}= & \frac{1}{512 \tau^{3}}\left(\frac{440423}{13513500}-\frac{4223}{17325} Y^{2}+\frac{47}{450} Y^{4}+\frac{6}{25} Y^{6}\right. \\
& \left.-\frac{13}{84} Y^{8}+\frac{211}{1575} Y^{10}-\frac{3}{154} Y^{12}\right)
\end{aligned}
$$

Using Eqn. 16, the steady-state components of $1 / \mathrm{Pe}_{3}$ and $1 / \mathrm{Pe}_{4}$ can be calculated:

$\frac{1}{P_{e_{3}}}=\frac{4}{17325} \times \frac{1}{\tau^{2}}$ and

$\frac{1}{P e_{4}}=-\frac{493}{123552000} \times \frac{1}{\tau^{3}}$

Compared with the steady-state part of $1 / \mathrm{Pe}_{2}$ (Eqn. 10), it is obvious that $1 / \mathrm{Pe}_{3}$ and $1 / \mathrm{Pe}_{4}$ can be neglected for most practical purposes, provided that $\tau$ is not very small. The latter condition is in fact not a real limitation because, as will be shown later, the theory developed above is valid only for $\tau$ values of the order of unity and greater. The higher order $1 / \mathrm{Pe}_{k}$ will decrease in magnitude further and their determination, although possible, is useless from a practical point of view. The results mentioned above show that the generalized axially dispersed plug flow model (Eqn. 7) can be reduced to the well known traditional axially dispersed plug flow model (Eqn. 6).

The calculation of $f_{k}$ is necessary not only for the determination of $\boldsymbol{P e}_{k}$ but also for the calculation of the concentration distribution in the $Y$ direction, provided that $C_{\mathrm{m}}(\theta, X)$ is known (Eqn. $3)$. In the region where Eqn. 18 is valid, $C_{\mathrm{m}}$ can be calculated using the axially dispersed plug flow model. This possibility gives considerable advantages in comparison with the straightforward
TABLE 2

Values of $f_{1}, f_{2}$ and $f_{3}$ for different $Y$ values

\begin{tabular}{llrr}
\hline$Y$ & \multicolumn{1}{l}{$f_{1}$} & \multicolumn{1}{l}{$f_{2}$} & \multicolumn{1}{l}{$f_{3}$} \\
\hline 0.0 & -0058333 & -0.000144 & 0000064 \\
0.1 & -0055846 & -0000193 & 0000059 \\
0.2 & -0048533 & -0000319 & 0.000045 \\
0.3 & -0.036846 & -0000466 & 0.000023 \\
04 & -0.021533 & -0.000558 & -0.000006 \\
0.5 & -0.003646 & -0.000517 & -0000036 \\
06 & 0015467 & -0.000294 & -0000063 \\
0.7 & 0034154 & 0.000106 & -0000076 \\
08 & 0050467 & 0000610 & -0.000060 \\
09 & 0.062154 & 0.001067 & 0.000011 \\
1.0 & 0.066667 & 0.001270 & 0.000182 \\
\hline
\end{tabular}

approach involving the solution of the convective-diffusion equation because the latter cannot be solved analytically whereas analytical solutions of the axially dispersed plug flow model have been obtained for various initial and boundary conditions. Even if for a given flow system the axially dispersed plug flow model cannot be solved explicitly, the corresponding numerical solution requires much less computations than that for the convective-diffusion equation. Comparison of the values of $f_{1}, f_{2}$ and $f_{3}$ for different $Y$ values (Table 2) shows that for practical purposes $f_{2}, f_{3}$ and higher order $f_{k}(k=4,5, \ldots)$ coefficients can be neglected.

Numerical solution of the convective-diffusion equation

The implicit alternating-direction finite-difference method [31] was used for the solution of Eqn. 2. It was chosen mainly for the following two reasons: stability limitations do not allow complete explicit differencing as required by the explicit method, although this method would have given the most straightforward and simple from calculational point of view solution of the problem, and the implicit method, which ensures both stability and convergence, leads to a system of linear algebraic equations each containing five unknowns, which requires a considerable amount of computation.

The implicit alternating-direction method avoids the disadvantages of both the explicit and the implicit method. The numerical integration is 
performed in two steps, each of duration equal to half of the time increment. First, the $X$ derivatives of the concentration (Eqn. 2) are implicitly differenced while the $Y$ derivatives are differenced explicitly. The second step gives an equation implicit in the $Y$ direction and explicit in the $X$ direction. In both steps the resulting system of algebraic equations has a tridiagonal coefficient matrix, thus allowing a straightforward solution based on the Gaussian elimination method [31].

The convergence and stability of the method cannot be analysed without actual calculations. For this reason, the size of the time and the two spatial increments was determined by trial and error. In most instances increments of $\theta$ and $X$ equal to 0.01 and 0.04 of $Y$ gave satisfactory results.

Equation 2 was solved originally for a stepfunction input. However, in flow-injection analysis the input signal is a rectangular pulse either in time (time injection) or in space (slug injection) [20]. It has been shown [32] that for obtaining the corresponding numerical solution it is not necessary to solve the equation in the case of step-function input twice (for the two boundaries of the sample plug, as was done earlier [33-37]). Provided that the numerical solution of Eqn. 2 in the case of a step function $\left(C_{\text {step }}\right)$ exists, the response of the system for slug $\left(C_{\text {slug }}\right)$ or time injection $\left(C_{\text {time }}\right)$ at $X=1$ can be obtained by the following simple relationships [32]:

$$
\begin{aligned}
& C_{\text {slug }}(\theta, 1, Y) \\
& \quad=C_{\text {step }}(\theta, 1, Y)-C_{\text {step }}(\theta, 1+\alpha, Y) \\
& C_{\text {tume }}(\theta, 1, Y) \\
& \quad=C_{\text {step }}(\theta, 1, Y)-C_{\text {step }}(\theta-\alpha, 1, Y)
\end{aligned}
$$

where $\alpha$ is the dimensionless length of the initial sample plug or the dimensionless duration of time injection.

In most instances the overall effect of the flow pattern on the concentration profile at the outlet of a given apparatus or at the point of detection (at a distance $L$ from the point of injection) is of substantial practical interest. For that reason, the average concentration in the cross-section of the flow at $X=1$ must be calculated:

$C_{\mathrm{m}}(\theta, 1)=\int_{0}^{1} C(\theta, 1, Y) \mathrm{d} Y$

Solution of the axially dispersed plug flow model

The solutions of the axially dispersed plug flow model (Eqn. 6) for the initial and boundary conditions of Eqn. 2 and for step- and rectangular-function input signal at $X=1$ are [32] for the step function

$C(\theta, 1)=\frac{1}{2} \operatorname{erfc}\left\{(1-\theta) /\left[2(\theta / P e)^{1 / 2}\right]\right\}$

and for the rectangular function

$$
\begin{aligned}
C(\theta, 1)= & \frac{1}{2}\left(\operatorname{erf}\left\{(\theta-1) /\left[2(\theta / P e)^{1 / 2}\right]\right\}\right. \\
& \left.+\operatorname{erf}\left\{(1+\alpha-\theta) /\left[2(\theta / P e)^{1 / 2}\right]\right\}\right)
\end{aligned}
$$

The solution of the axially dispersed plug flow model is indifferent to the type of injection [32], 1.e., the solutions for time and slug injection are identical, provided that $\alpha$ is the same.

The calculation of $C(\theta, 1)$ for the case of rectangular-function input can be speeded up if, instead of calculating twice the error function in Eqn. 27 for each $\theta$ value, the step-function solution is used. This approach requires only one calculation of the error function $[\operatorname{erfc}(z)=1-$ $\operatorname{erf}(z)]$ in combination with Eqn. 24.

\section{Software}

The method for numerical integration of Eqn. 2 outlined above and the processing of the numerical data in the framework of the axially dispersed plug flow model were programmed in $\mathrm{C}$ and run on an IBM PC compatible computer.

\section{RESULTS AND DISCUSSION}

Solutions of the convective-diffusion equation were obtained for Fourier numbers $(\tau)$ varying in the range $0.2-3.0$ and $\beta$ values in the range 0 
$1.562 \times 10^{-2}$. All parallel plate flow systems used in artificial kidneys and flow-through analytical manifolds are within the $\tau-\beta$ region defined above.

\section{Influence of the geometrical group $\beta$}

It should be taken into consideration that for $\beta=0$ either the height of the channel is infinitesimally small or the axial molecular diffusion can be neglected, because $\beta$ is the coefficient of $\partial^{2} C / \partial X_{1}^{2}$ (Eqn. 2).

The numerical results showed that for $\beta \leqslant$ $1.562 \times 10^{-4}$ in the Fourier number range mentioned above this geometrical group did not affect the solution of Eqn. 2. Only for $\beta=1.562 \times 10^{-2}$ was a slight deviation observed from the solution obtained for $\beta=0$. Taking into consideration that if the characteristic length is assumed to be equal to $2 \mathrm{~m}$ then $\beta=1.562 \times 10^{-2}$ will correspond to $a=0.25 \mathrm{~m}$ or to a channel height equal to $0.5 \mathrm{~m}$. Such channel dimensions will hardly be encountered in any real process heat or mass exchanger. As far as haemodialysers or analytical flowthrough dialysers are concerned, it can be said that $\beta$ is always several orders of magnitude less than the value given above. Obviously in all instances of practical interest $\beta$ does not affect the concentration distribution and for that reason Eqn. 2 can be simplified to some extent by neglecting the axial diffusion term, i.e., $\tau \beta\left(\partial^{2} C / \partial X_{1}^{2}\right)=0$.

In all subsequent calculations $\beta$ will be assumed to be equal to 0 .

\section{Influence of the Fourier number}

The Fourier number, which is the "reduced to molecular diffusion scale mean residence time" of the flow system, is its most important parameter, determining almost solely the concentration distribution. For that reason it will be used as a criterion for the applicability of the axially dispersed plug flow model for the description of the dispersion in laminar parallel plate flow.

In Fig. 2a, $C_{\mathrm{m}}(\theta, 1)$ curves calculated by the convective-diffusion equation (Eqn. 2 ) and by the axially dispersed plug flow model with $P e=52.5 \tau$ (Eqn. 18) for step-function concentration input are compared. It can be seen that for $\tau \geqslant 1.0$ the responses of the system calculated in both ways
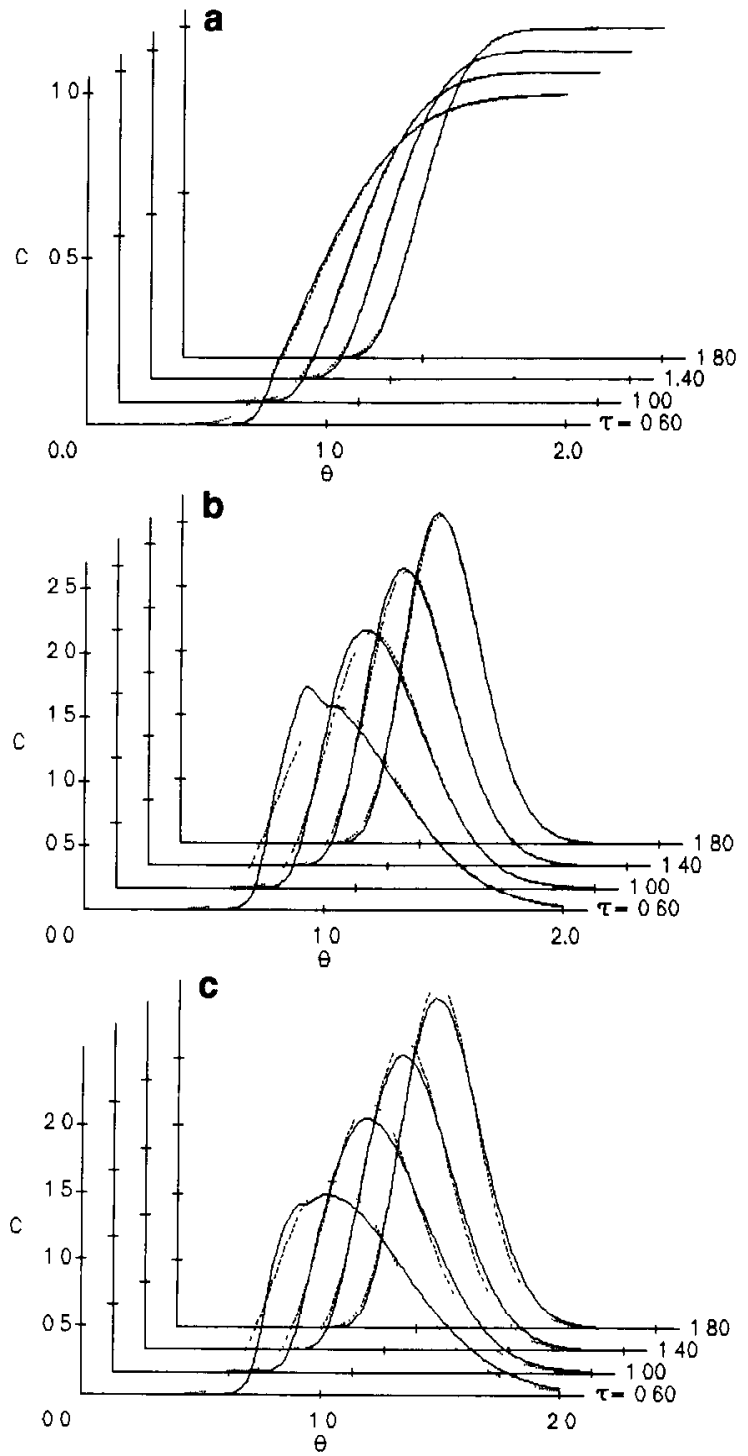

Fig. 2 Concentration-tıme curves at $X=1$ calculated by Eqn 2 (solid lines) and Eqn. 18 (dotted lines) for (a) step-function concentration injection, (b) time injection ( $\alpha=0.2)$ and (c) slug injection $(\alpha=0.2)$

are very close to each other. The same can be observed with time injection $(\alpha=0.2)$ (Fig. 2b). If slug injection is used the deviation between $C_{\mathrm{m}}(\theta, 1)$ calculated by Eqns. 2 and 6 is more pronounced (Fig. 2c) than in the case of time injection (Fig. 2b). This is due to the differences in the dispersion of the initial sample plug during the 
period of its introduction into the system, i.e., $0 \leqslant \theta \leqslant \alpha$. In slug injection this is the period during which the initial sample slug will leave the injection section, provided that it moves with the average flow rate $(u)$. After this period the dispersion process in both instances proceeds in the same way. For that reason it is important to compare the concentration distribution at the end of the injection period. During this period the dispersion process is practically governed only by the convection and for the calculation of the concentration distribution all terms in Eqn. 2 containing $\tau$ can be neglected. The results obtained are very similar to those for tubular flow presented by Reijn et al. (Fig. 2 in [20]). It can be seen that at the end of the injection period (i.e., $\theta=\alpha$ ) the sample slug is broader with slug injection than with time injection. This will lead in the former instance to the formation of a lower and broader concentration-time peak at $X=1$ than in the latter. In practice, the sample injection is usually performed by a valve. This means that at $\theta=0$ the flow starts to accelerate from a motionless state to that of steady-state motion. Although the transitional period before the establishment of fully developed laminar flow is much shorter than the mean residence time of the flow system [38], it may include a substantial part of the injection period. In such an event it can be expected that the trailing boundary of the sample plug in the $Y$ direction will have a flat rather than a parabolic shape. For this reason it seems that time injection, which assumes a constant concentration at $X=0$ during the injection period, is a better approximation of the real physical picture than slug injection.

The results in Fig. 2a and $b$ show that for $\tau \geqslant 1.0$ the axially dispersed plug flow model with $P e=52.5 \tau$ can be used for the calculation of $C_{\mathrm{m}}(\theta, 1)$.

The hydraulic model mentioned above assumes a homogeneous concentration in the $Y$ direction. From this point of view it will be interesting to see how the Fourier number affects the concentration distribution in the $Y$ direction. In Fig. 3 this dependence can be observed. As could be expected, with increasing $\tau$ value the concentration gradient in the $Y$ direction decreases. For $\tau \geqslant 1.0$
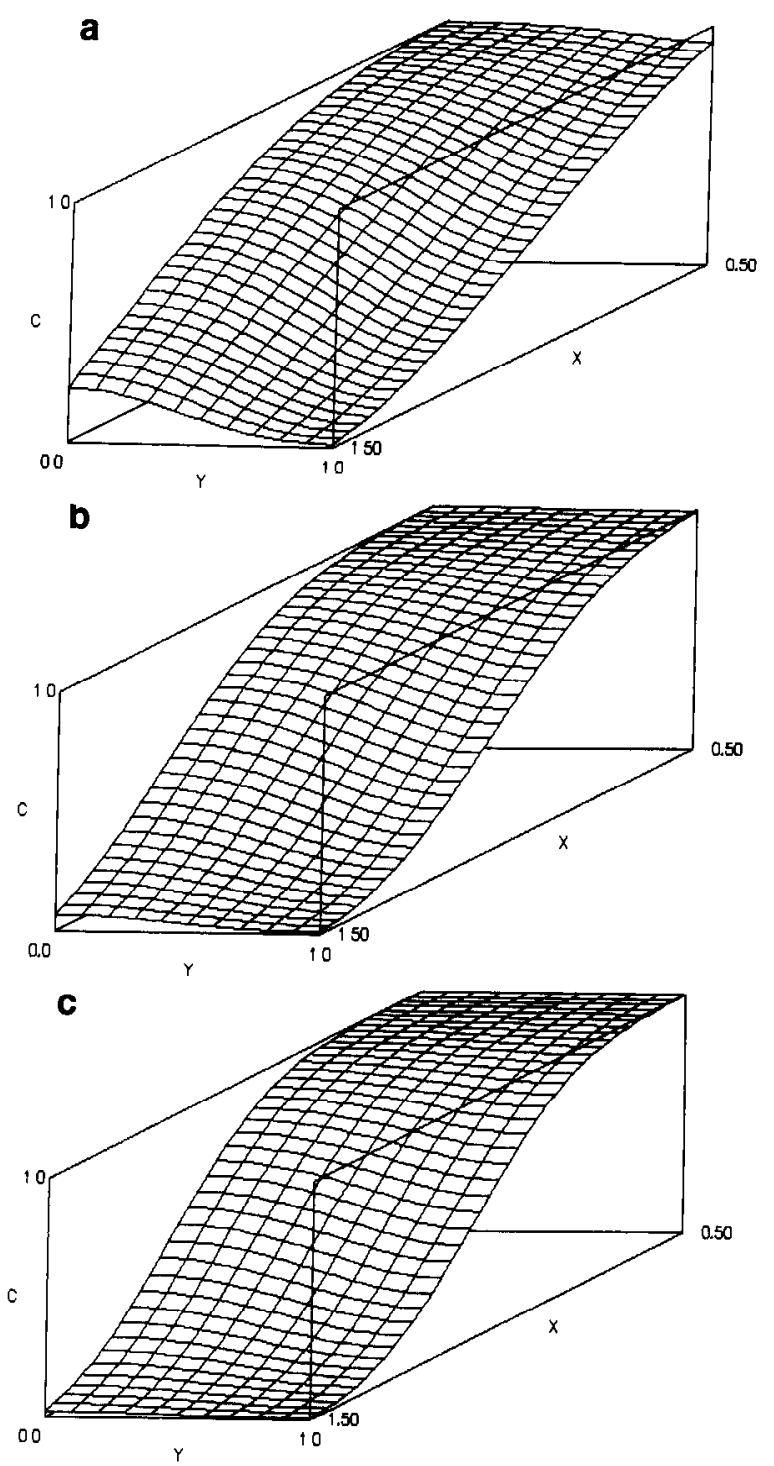

Fig 3. Concentration distribution at $\theta=1$ calculated by Eqn. 2 for Founer numbers $(\tau)=$ (a) 0.5, (b) 10 and (c) 1.5 .

the $Y$ distribution can be calculated on the basis of Eqns. 3 and 26 neglecting all but the first term $\left(f_{1} \partial C_{\mathrm{m}} / \partial X_{1}\right)$ in the sum. Taking into consideration that

$\mathrm{d} C_{\mathrm{m}} / \mathrm{d} X=\frac{1}{2} \sqrt{P e / \pi \theta} \exp \left[-P e(X-\theta)^{2} / 4 \theta\right]$ 


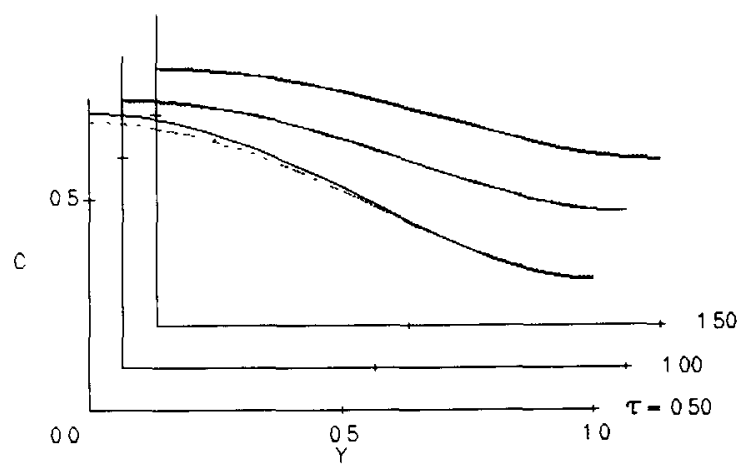

Fig 4. Concentration distribution in the $Y$ direction at $X=1$ and $\theta=1$ calculated by Eqn. 2 (solid lines) and Eqn 29 (dotted lines) for Founer numbers $(\tau)=0.5,1.0$ and 15

it can be written

$$
\begin{aligned}
C(\theta, X, Y) \\
=\frac{1}{2}\left(\operatorname{erfc}\left\{(X-\theta) /\left[2(\theta / P e)^{1 / 2}\right]\right\}\right. \\
\quad-\frac{1}{4 \tau}\left(-\frac{7}{30}+Y^{2}-\frac{Y^{2}}{2}\right) \times \sqrt{P e / \pi \theta} \\
\left.\quad \times \exp \left[-P e(X-\theta)^{2} / 4 \theta\right]\right)
\end{aligned}
$$

Figure 4 illustrates the agreement between the $C(\theta, 1, Y)$ calculated by Eqns. 2 and 29 for different Fourier numbers. Obviously for $\tau \geqslant 1.0$. Eqn. 29 gives results that are acceptable from practical point of view.

\section{Conslusions}

A theoretical relationship for calculating the Peclet number for fully developed parallel plate laminar flow was derived. Its applicability for real flow systems was determined on the basis of numerical solution of the convective-diffusion equation. It was found that for laminar parallel plate flow systems encountered in mass- and heat-exchange devices in industry, medicine and chemical analysis the axial diffusion can be neglected. In most instances of practical interest the Peclet number can be considered to be independent of time and equal to $52.5 \tau$. The theory developed here allows the calculation of the concentration distribution in the $Y$ direction using the solution of the axially dispersed plug flow model.
The results obtained can be used for the development of mathematical models of devices utilizing parallel plate laminar flow (e.g., industrial parallel plate heat and mass exchangers, artificial kidneys and flow-injection manifolds with on-line dialysis modules).

\section{REFERENCES}

1 R B Bird, W.E. Stewart and E N. Lightfoot, Transport Phenomena, Wiley, New York, 1960

2 J G. Knudsen and D.L Katz, Fluid Dynamics and Heat Transfer, McGraw-Hill, New York, 1958.

3 L Grumsrud and A.L Babb, AIChE Symp. Ser , 62 (1966) 20,66

4 C.K Colton, K.A. Smith, P Strove and E.W Merrill, AIChE J., 17 (1971) 773.

5 J.M Kooljman, Chem Eng. Sc1, 28 (1973) 1149

6 D O. Cooney, S -S. Kım and E.J Davis, Chem Eng. Sc1., 29 (1974) 1731

7 D.O Cooney, E.J Davıs and S.-S. Kım, Chem. Eng J., 8 (1974) 213

8 L.T Skeggs, Jr., Am. J Clin Pathol., 28 (1957) 311.

9 A.H Kadish and D.A. Hall, Clin. Chem, 11c (1965) 869.

10 E.H Hansen and J Ruzicka, Anal. Chim Acta, 87 (1976) 353

11 W E. van der Linden, Anal Chım. Acta, 151 (1983) 359.

12 B Bernhardsson, E Martıns and G. Johansson, Anal. Chım. Acta, 167 (1985) 111.

13 L. Risinger, G Johansson and T. Thorneman, Anal Chım Acta. 224 (1989) 13

14 D.M Himmelblau and K B. Bischoff, Process Analysis and Simulation. Determinıstic Systems, Wiley, New York, 1968

$15 \mathrm{O}$ Levenspiel and K.B. B1schoff, Adv Chem. Eng., 4 (1963) 95.

16 J. Ruzicka and EH Hansen, Flow Injection Analysis, Wiley, New York, 2nd edn., 1988.

17 M Valcarcel and M.D. Luque de Castro, Flow-Injection Analysis Principles and Applications, Horwood, Chichester, 1987.

19 W E. van der Linden, in J.L. Burguera (Ed.), Flow Injection Atomic Spectroscopy, Dekker, New York, 1989.

20 J.M Reijn, W.E. van der Linden and $H$ Poppe, Anal Chım Acta, 114 (1980) 105

$21 \mathrm{~J} M$ Reıjn, W E. van der Linden and H. Poppe, Anal. Chım Acta, 126 (1981) 1

22 S D. Kolev and E. Pungor, Anal Chem , 60 (1988) 1700.

23 S.D Kolev and E. Pungor, Anal Chim. Acta, 208 (1988) 117.

24 S D. Kolev and E. Pungor, Anal. Chım Acta, 208 (1988) 133

25 G. Taylor, Proc R Soc London, Ser. A, 219 (1953) 186

26 G Taylor, Proc R Soc. London, Ser A, 225 (1954) 231

27 R. Ans, Proc. R Soc. London, Ser. A, 235 (1956) 67 
28 W.N. Gill, Proc R Soc London, Ser. A, 298 (1967) 335

29 W.N Gill, Proc. R. Soc. London, Ser. A, 316 (1970) 341.

30 V. Ananthakrishnan, W N. Gill and A J Barduhn, AIChE J., 11 (1965) 1063.

31 B. Carnahan, H A. Luther and J.O. Wilkes, Applied Numencal Methods, Wiley, New York, 1969

32 S.D. Kolev, Anal Chim Acta, 229 (1990) 183.

33 H. Bate, S. Rowlands and J A. Sirs, J. Appl Physiol, 34 (1973) 866

34 J.T. Vanderslice, K.K. Stewart, A G Rosenfeld and D.J. Higgs, Talanta, 28 (1981) 11
35 J T. Vanderslice, A G Rosenfeld and G.R. Beecher, Anal. Chim Acta, 179 (1986) 119.

$36 \mathrm{C} \mathrm{C}$ Painton and H.A. Mottola, Anal. Chim Acta, 158 (1984) 67

37 H. Wada, S. Hiraoka, Y Yuchı and G Nakagawa, Anal. Chım Acta, 179 (1986) 181.

38 S D. Kolev and E Pungor, Anal. Chim Acta, 201 (1987) 109 